\title{
3D Fast Factorized Back-Projection in Cartesian Coordinates
}

\author{
Juliana A. Góes ${ }^{1}$, Valquiria Castro ${ }^{1}$, Leonardo Sant'Anna Bins ${ }^{2}$, Hugo E. Hernandez-Figueroa ${ }^{1}$ \\ ${ }^{1}$ School of Electrical and Computer Engineering, University of Campinas - UNICAMP, Campinas, Brazil \\ ${ }^{2}$ National Institute for Space Research - INPE, São José dos Campos, Brazil \\ Email: juliana.goes@engineer.com
}

\begin{abstract}
This paper presents a novel 3D Fast Factorized Back-Projection (FFBP) algorithm that is based on an extension of the quadtree approach. A flexible 3D tree structure is generated from a modified Morton order or Z-order curve, a recursive space-filling curve that is suitable for FFBP algorithms. This paper presents, as well, an original method for defining sub-apertures. The proposed algorithm can be applied to any flight path and is about $90 \%$ faster than the direct backprojection, yielding high-resolution 3D images with low phase errors and high degrees of coherence.
\end{abstract}

Keywords—SAR, radar, back-projection, FFBP, 3D imaging.

\section{INTRODUCTION}

In recent years, the scientific community has seen a growing interest in Synthetic Aperture Radar (SAR) systems carried by drones. This rising technology could support a variety of flight trajectories for many applications, including tomographic imaging $[1,2]$. Therefore, a suitable processing algorithm would be the time domain Back-Projection (BP), which provides good focusing quality with arbitrary flight paths. However, the main disadvantage of the BP algorithm is its high computational burden. Fortunately, Fast BP algorithms have been developed to reduce processing time.

In 1996, McCorkle and Rofheart [3] proposed a solution based on a divide-and-conquer strategy that results in a quadtree data structure. The root node represents the original SAR data that covers the whole imaging area. At each recursion, parent sub-apertures are combined to form progressively longer children sub-apertures, as though building antenna-array beam patterns that cover increasingly smaller sub-images with each recursion. The phase center of each child sub-aperture is defined by the weighted mean position of all parent phase centers that comprise it.

In 2003, Ulander et al. [4] presented the Fast Factorized Back-Projection (FFBP) algorithm. Instead of applying cartesian coordinates, it represents image data in local polar coordinates to reduce the number of operations. In addition, that algorithm simplifies calculations by assuming a linear flight path. In this way, it is applicable for ultrawideband SAR systems, for which it can deal with significant deviations from a linear trajectory.

These methods can also be applied to circular trajectories, provided that some modifications are introduced. In [5], the circular flight track is approximated by equilateral polygons, and then the FFBP algorithm [4] is adjusted to this new configuration. In [6], the sub-apertures are defined by

This study was financed in part by the Coordenação de Aperfeiçoamento de Pessoal de Nível Superior - Brasil (CAPES) - Finance Code 001; and the São Paulo Research Foundation (FAPESP) - PITE 2017/19416-3 and PIPE 2018/00601-8. weighted mean positions, as in [3], but the phase centers are described in polar coordinates instead of cartesian coordinates.

Recently, the FFBP algorithm has been extended to 3D by using a set of conversions between sub-apertures whose data are represented in spherical coordinates [7]. That algorithm was designed for downward-looking sonar systems that move along approximately linear paths. Although it achieves a significant reduction in processing time, an adaptation to arbitrary trajectories would not be straightforward.

This work presents a 3D FFBP algorithm that was developed through an extension of the quadtree structure, in cartesian coordinates, using a modified Morton order curve [8] to perform data mapping. To the best of our knowledge, the method for defining sub-apertures introduced in this work is an original one. The proposed algorithm is applicable for any flight paths.

\section{3D FAST FACTORIZED BACK-PROJECTION}

The 3D FFBP presented in this paper is a modification of the McCorkle and Rofheart's algorithm [3], with the alterations introduced henceforth. In particular, the 3D subimages are created by employing a modified Morton order curve [8], and the sub-apertures are defined with a unique method based on data mapping.

\section{A. Generation of $3 D$ sub-images}

For a 3D FFBP, the most evident extension of the quadtree structure would be an octree. One solution for organizing data in a quadtree structure that can be readily expanded to an octree is the Morton order curve [8,9]. It was initially created for mapping $2 \mathrm{D}$ information in $1 \mathrm{D}$ data, and it is built by recursively dividing one square into four squares, arranging them in a Z-shaped manner, that is why it is also known as the $Z$-order curve. Thanks to this recursive construction, it can be easily applied to FFBP algorithms.

Furthermore, SAR images often have non-uniform resolutions. Even SAR Tomography techniques that provide high-resolution 3D images achieve better resolutions in the horizontal $(x, y)$ plane than in the vertical $z$-direction $[10,11]$. Hence, more flexible tree structures could be appealing for 3D SAR applications.

This work proposes a modified Morton order curve that divides each parent sub-image into $D_{p x} \times D_{p y} \times D_{p z}$ child subimages, where $D_{p x}, D_{p y}$ and $D_{p z}$ are the number of divisions in the $x$-, $y$ - and $z$-directions, respectively. Fig. 1 shows the first and second recursions for the modified Morton order curve with a $3 \times 3 \times 2$ partition. 


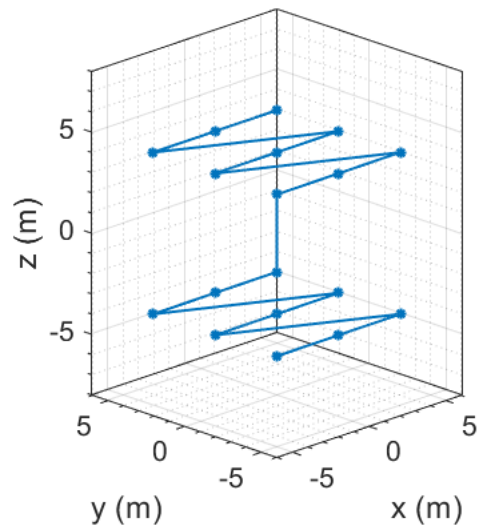

(a)

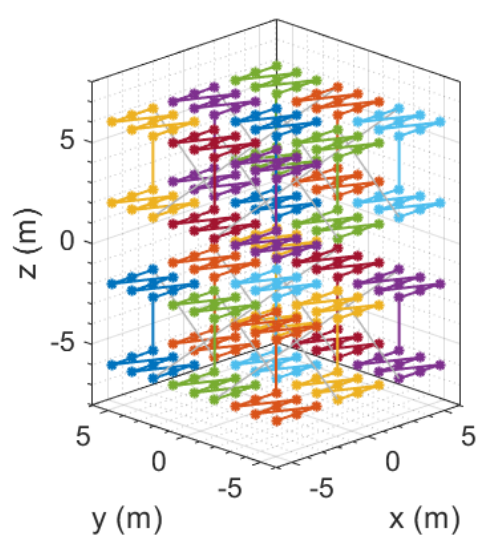

(d)

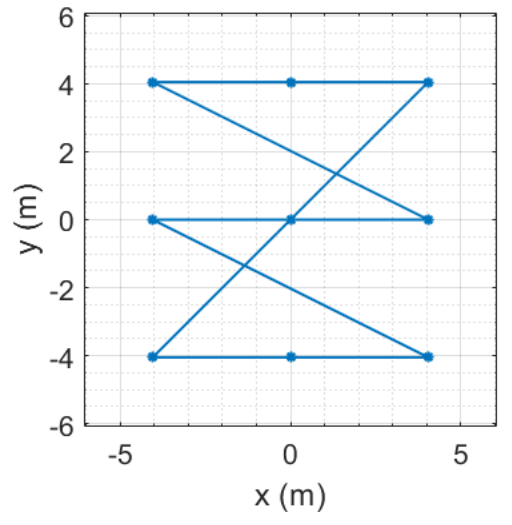

(b)

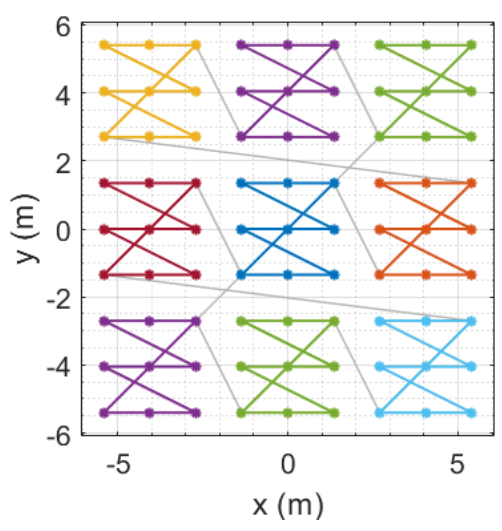

(e)

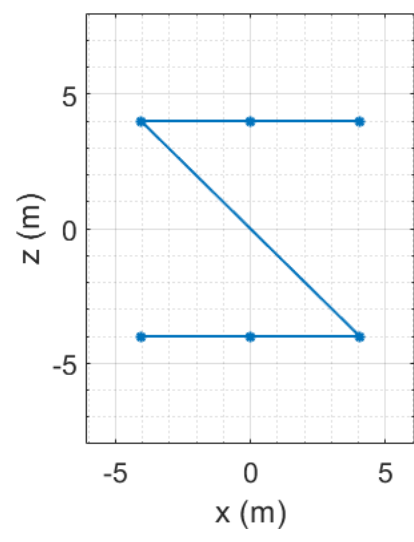

(c)

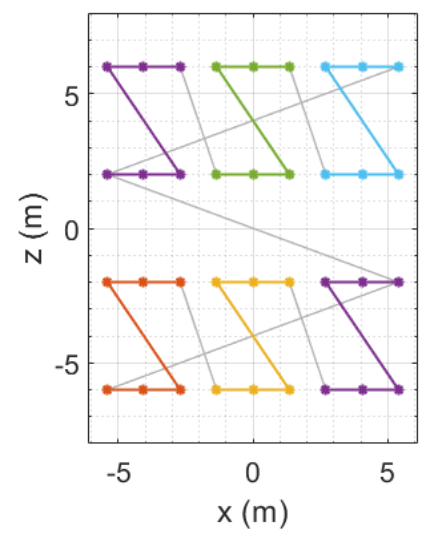

(f)

Fig. 1. The modified Morton order curve with a $3 \times 3 \times 2$ partition: (a-c) the first recursion and (d-f) the second recursion. The columns (a,d), (b,e) and (c,f) show, respectively, perspective, top and front views.

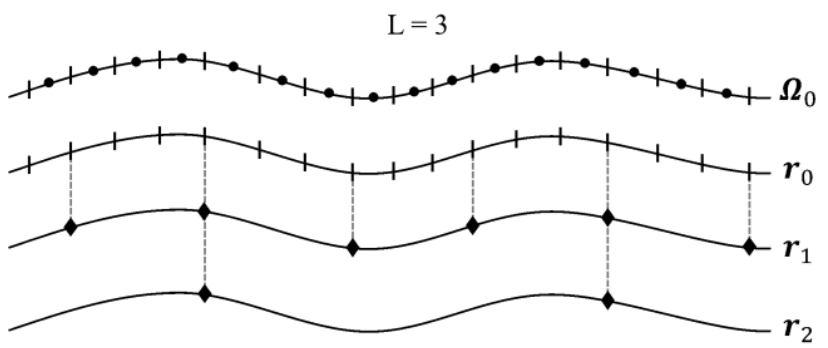

(a)

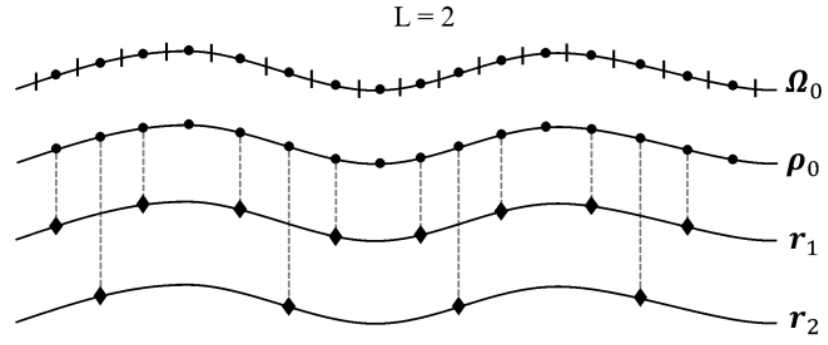

(b)

Fig. 2. Definition of sub-apertures with (a) $L=3$ and (b) $L=2$. Dashes represent the original SAR positions $\left(\boldsymbol{r}_{0}\right)$, circles indicate the midpoints ( $\left.\boldsymbol{\rho}_{0}\right)$ between two consecutive SAR positions, and diamonds symbolize the sub-aperture phase centers $\left(\boldsymbol{r}_{\mathrm{c}}\right)$.

At the end of the 3D FFBP algorithm, this 1D data is mapped back into 3D information by using recurrent sequences. Let $D_{p}=D_{p x} \times D_{p y} \times D_{p z}$ be the total number of divisions at each recursion and let $q_{x}$ be the sequence associated with the $x$-direction, thus:

$$
\begin{gathered}
q_{x}[0]=0 \\
q_{x}\left[n D_{p x}+i\right]=D_{p} q_{x}[n]+i
\end{gathered}
$$

where $i=0, \ldots, D_{p x}-1$. The sequences related to the $y$ - and $z$ directions, namely $q_{y}$ and $q_{z}$, are created using a similar procedure.
Then, the $3 \mathrm{D}$ indexing $I_{3 D}$ is obtained from:

$$
I_{3 D}=q_{x}+D_{p x} q_{y}+D_{p x} D_{p y} q_{z}
$$

The choice $D_{p x}=D_{p y}=2$ and $D_{p z}=1$ produces the original Morton order curve.

\section{B. Definition of sub-apertures}

The proposed method for defining sub-apertures is illustrated in Fig. 2. It is based on data mapping and does not depend on the flight path geometry. Let $\boldsymbol{r}_{0}$ be the array of the original SAR positions (at the root node) and let $L$ be the number of parent sub-apertures that are combined to form a child sub-aperture at each iteration. There are two particular 
cases and a general case for defining $\boldsymbol{r}_{\mathrm{c}}$, the array of phase centers in the child node $c$.

1) $L$ is an odd number: When $L$ is odd, the sub-aperture phase centers $\boldsymbol{r}_{c}$ are always a subset of the original SAR positions $\boldsymbol{r}_{0}$, as depicted in Fig. 2(a). What is more, if $\boldsymbol{r}_{p}$ is the array of parent phase centers corresponding to the previous node, then $\boldsymbol{r}_{c}$ is also a subset of $\boldsymbol{r}_{p}$. Therefore, either of the following expressions can be used:

$$
\begin{gathered}
\boldsymbol{r}_{c}[k]=\boldsymbol{r}_{0}\left[\left(L^{c}-1\right) / 2+k L^{c}\right] \\
\boldsymbol{r}_{c}[k]=\boldsymbol{r}_{p}[(L-1) / 2+k L]
\end{gathered}
$$

where $k=0, \ldots, N_{c}-1$, and $N_{c}$ is the number of aperture elements in the child node.

2) $L$ is a power of 2: Let $\boldsymbol{\rho}_{0}$ be an array composed of all the points that fall halfway between two successive SAR positions in $\boldsymbol{r}_{0}$. When $L$ is a power of 2 , the set of sub-aperture phase centers $\boldsymbol{r}_{c}$ is always a subset of $\boldsymbol{\rho}_{0}$, as seen in Fig. 2(b). Then, $\boldsymbol{r}_{c}$ can be expressed as:

$$
\boldsymbol{r}_{c}[k]=\boldsymbol{\rho}_{0}\left[L^{c} / 2-1+k L^{c}\right]
$$

where $k=0, \ldots, N_{c}-1$. In this way, the calculation of mean positions is only performed in the root node, when deviation errors are the least significant.

3) General case: Let $\boldsymbol{\Omega}_{0}$ be the interleaved combination of both $\boldsymbol{r}_{0}$ and $\boldsymbol{\rho}_{0}$ (see Fig. 2). So, for any value of $L$, the set of sub-aperture phase centers $\boldsymbol{r}_{c}$ is always a subset of $\boldsymbol{\Omega}_{0}$ :

$$
\boldsymbol{r}_{c}[k]=\boldsymbol{\Omega}_{0}\left[L^{c}-1+2 k L^{c}\right]
$$

where $k=0, \ldots, N_{c}-1$. In practice, $\boldsymbol{\Omega}_{0}$ can be obtained directly from $\boldsymbol{r}_{0}$ using an interpolation function. Again, this interpolation is only performed in the root node.

\section{Other modifications}

Just as in [3], at a given iteration, range samples are taken along the lines that connect each child phase center $\boldsymbol{r}_{c}[k]$ to the center of each child sub-image. Though, unlike [3], the number of range bins is constant for each node. The reason is to ensure full coverage, so all samples within the sphere that circumscribes the 3D image are considered. In [6], full coverage is achieved by calculating distances on the diagonals of the sub-images, but then $2 \mathrm{D}$ (or 3D, in this case) interpolations would be required for combining subapertures.

Finally, to ensure the coherent summation of sub-aperture data for any flight trajectory, we included the phase compensation term presented in [12], thus reducing artifacts and improving image quality. Thereby, the child radar data $s_{c}$ is generated from the parent radar data $s_{p}$ as follows $[3,12]$ :

$$
s_{c}[k, m]=\sum_{l \in \Lambda_{p, k}} s_{p}\left[l, v_{c, k, l, m}\right] \Delta \Phi_{c, k, l, m}
$$

$$
\Delta \Phi_{c, k, l, m}=\exp \left\{-j \frac{4 \pi}{\lambda_{0}}\left(\Psi_{c, k, m}-\Gamma_{c, k, l, m}\right)\right\}
$$

where $\lambda_{0}$ is the carrier wavelength; $c, p, k, l$ and $m$ indicate, respectively, a child node, a parent node, a child sub-aperture, a parent sub-aperture, and the range sample. $\Psi_{c, k, m}$ is the range from the child phase center $\boldsymbol{r}_{c}[k]$ to the $m^{\text {th }}$ range bin in $s_{c}[k, m]$; whereas $\Gamma_{c, k, l, m}$ is the range from the parent phase center $\boldsymbol{r}_{p}[l]$ to that same range bin. The floating-point index $v_{c, k, l, m}$ is a function of $\Gamma_{c, k, l, m}$ and the values for $s_{p}\left[l, v_{c, k, l, m}\right]$ are calculated with linear interpolation. The index set $\Lambda_{c, k}$ lists which parent sub-apertures compose the $k^{\text {th }}$ child subaperture.

\section{Simulation Results}

\section{A. Point spread function}

The first simulated scenario consists of a drone-borne SAR system, like the one presented in $[1,13]$, operating at Pband, and performing a helical trajectory with constant radius and constant speed, as depicted in Fig. 3. There are nine scatterers: one at the origin of an East-North-Up system, and the others at the vertices of a cube with sides $8 \mathrm{~m}$ long, centered at the origin. Table 1 shows the simulated radar acquisition parameters.

TABLE I. SIMULATED RADAR ACQUISITION PARAMETERS

\begin{tabular}{|l|c|c|}
\hline \multicolumn{1}{|c|}{ Parameters } & Values & Units \\
\hline Carrier wavelength & 0.75 & $\mathrm{~m}$ \\
\hline Bandwidth & 150 & $\mathrm{MHz}$ \\
\hline Range resolution & 1 & $\mathrm{~m}$ \\
\hline Pulse repetition frequency & 200 & $\mathrm{~Hz}$ \\
\hline Flight path radius & 180 & $\mathrm{~m}$ \\
\hline Height at the top of the flight path & 120 & $\mathrm{~m}$ \\
\hline Height at the bottom of the flight path & 80 & $\mathrm{~m}$ \\
\hline Number of helical turns & 5 & - \\
\hline Radar speed & 6.5 & $\mathrm{~m} / \mathrm{s}$ \\
\hline
\end{tabular}

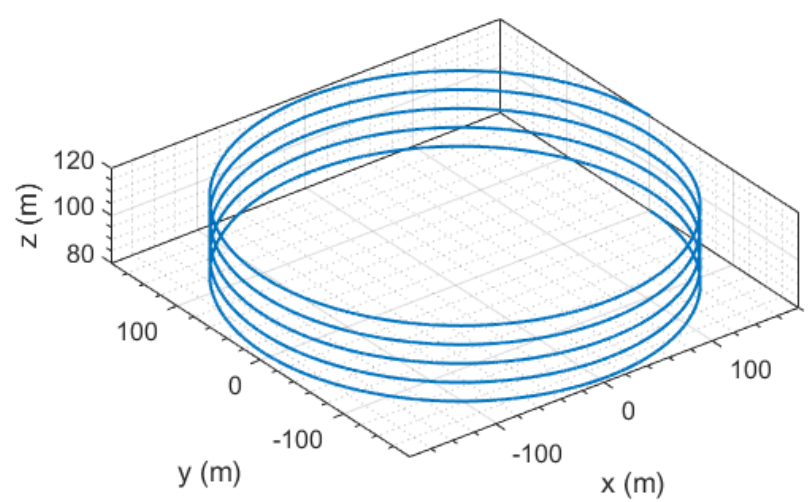

Fig. 3. Simulated helical flight path.

The 3D FFBP is setup with six iterations in which the subapertures are combined in groups of $L=3$, and the range sampling is $0.125 \mathrm{~m}$. At the root node, there are 174,960 
$\left(3^{6} \times 240\right)$ aperture positions, and the imaged volume is $12.15 \times 12.15 \times 14.4 \mathrm{~m}^{3}$. The first iteration builds the initial sub-image; the following four iterations perform $3 \times 3 \times 2$ partitions, as shown in Fig. 1; and the last iteration divides each sub-image into $3 \times 3 \times 3$ smaller ones. Finally, the remaining 240 data blocks are coherently combined.

The $3 \mathrm{D}$ output image has $243 \times 243 \times 48$ voxels of dimension $0.05 \times 0.05 \times 0.3 \mathrm{~m}^{3}$. Fig. 4(a) shows the distribution of all nine scatterers after processing with the $3 \mathrm{D}$ FFBP and Fig. 4(b) shows a closer caption of the central scatterer. The opaque red curves are $-3 \mathrm{~dB}$ isosurfaces, and the translucent yellow ones are $-13 \mathrm{~dB}$ isosurfaces.

Fig. 5 displays the point spread function for the 3D FFBP and the BP algorithms. It can be seen that the plot curves are nearly the same for both algorithms. The $(x, y)$ plane has a refined resolution of $0.16 \mathrm{~m}$, but a poor peak-to-sidelobe ratio (PSLR) of $-9.1 \mathrm{~dB}$, whereas the $z$-direction presents a coarser resolution of $1.53 \mathrm{~m}$ with a better PSLR of $-28.7 \mathrm{~dB}$. Furthermore, the mean phase error is $10^{-4} \mathrm{rad}$, and the standard deviation is $0.12 \mathrm{rad}$, which is more than three times lower than the recommended threshold of $\pi / 8 \mathrm{rad}$ [4]. The mean and the standard deviation of the magnitude error are, respectively, $0.1 \mathrm{~dB}$ and $0.9 \mathrm{~dB}$. The degree of coherence between the resulting images from the $3 \mathrm{D}$ FFBP and the BP algorithm is 0.9993 . If the two images were entirely identical, this parameter would be equal to one [14]. Table 2 summarizes these results.

TABLE II. POINT SPREAD FUnCTION - SimUlation RESUlts

\begin{tabular}{|l|c|c|}
\hline \multicolumn{1}{|c|}{ Parameters } & Values & Units \\
\hline 3 dB resolution in the $(x, y)$ plane & 0.16 & $\mathrm{~m}$ \\
\hline 3 dB resolution in the $z$-direction & 1.53 & $\mathrm{~m}$ \\
\hline PSLR in the $(x, y)$ plane & -9.1 & $\mathrm{~dB}$ \\
\hline PSLR in the $z$-direction & -28.7 & $\mathrm{~dB}$ \\
\hline Mean phase error & $10^{-4}$ & $\mathrm{rad}$ \\
\hline Standard deviation of the phase error & 0.12 & $\mathrm{rad}$ \\
\hline Mean magnitude error & 0.1 & $\mathrm{~dB}$ \\
\hline Standard deviation of the magnitude error & 0.9 & $\mathrm{~dB}$ \\
\hline Degree of coherence & 0.9993 & - \\
\hline Processing time $-3 \mathrm{D}$ FFBP & 3.45 & $\mathrm{~h}$ \\
\hline Processing time - BP & 39.09 & $\mathrm{~h}$ \\
\hline
\end{tabular}

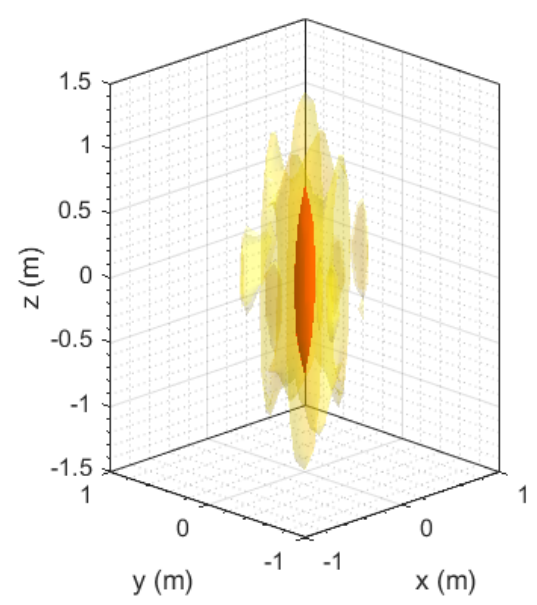

(b)

Fig. 4. The 3D output image processed with the 3D FFBP: (a) $-3 \mathrm{~dB}$ isosurfaces for the entire imaged volume; (b) $-3 \mathrm{~dB}$ isosurface in opaque red and -13 $\mathrm{dB}$ isosurfaces in translucent yellow for the target at the origin.

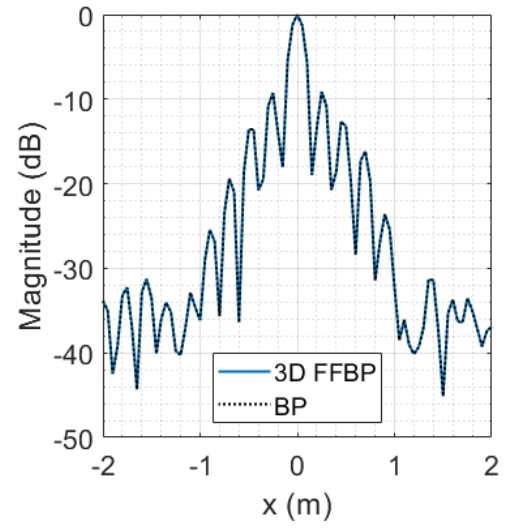

(a)

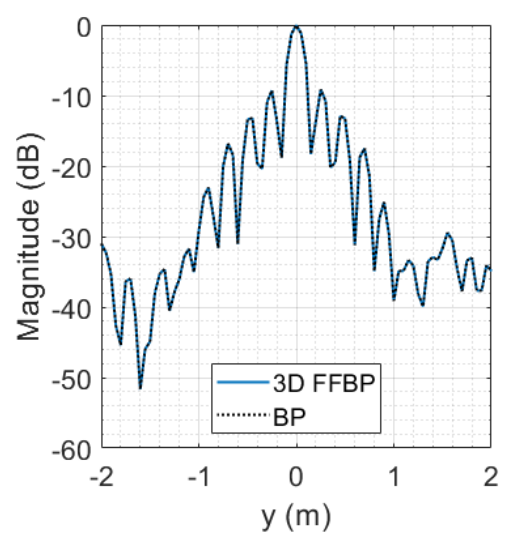

(b)

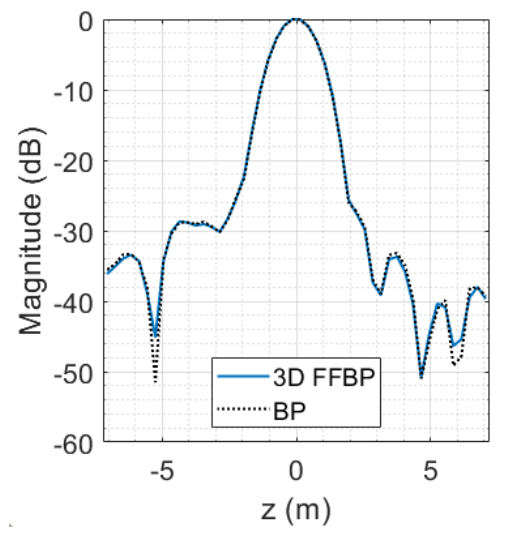

(c)

Fig. 5. Comparison between the 3D FFBP and the BP algorithms. Point spread function: normalized magnitude in dB vs. (a) $x$, (b) $y$, and (c) $z$. 
The low phase error is due, in part, to the choice of the sub-images dimensions, as the analysis provided by Ulander et al. [4] suggests. However, without (9), the results would be far worse. The standard deviation of the phase error would be $1.63 \mathrm{rad}$, yielding a coherence of 0.29 .

All algorithms were written in MATLAB R2018a without applying any parallel computing functions. All simulations were executed on an Intel(R) Core(TM) i7-7700 CPU (3.60 $\mathrm{GHz}$ ) with $64 \mathrm{~GB}$ RAM. The processing time with the $3 \mathrm{D}$ FFBP was 3.45 hours ( $\sim 0.14$ days), while the BP took 39.09 hours $(\sim 1.6$ days $)$. This difference represents a reduction of 11.3 times $(91.1 \%)$ in computation time. This result concerns the 3D FFBP employing the general case (7), which was 5.5 minutes faster than using (5), and 19.1 minutes faster than adopting the usual method of calculating weighted mean positions. The RAM usage did not exceed $65 \%$ in any of the simulations.

\section{B. Random point scatterers}

The second simulated scenario also uses the helical flight path depicted in Fig. 3 with the radar acquisition parameters of Table 1, but a random phase error is added to the simulated radar data to represent motion data inaccuracies. The total relative position error of a drone-borne SAR system was measured in [13] and had a standard deviation of $7.4 \mathrm{~mm}$, which corresponds to a phase error standard deviation of 0.12 rad at the $0.75 \mathrm{~m}$ wavelength. Thus, the simulated radar data is perturbed by adding a normally distributed phase error with zero mean and the standard deviation calculated above.

Real radar cross-section data can be modeled as a distribution of ideal isotropic point scatterers [15]. For that reason, a random distribution of point scatterers is adopted for this scenario. The initial target grid has $81 \times 81 \times 9$ points with $0.15 \mathrm{~m} \times 0.15 \mathrm{~m} \times 1.5 \mathrm{~m}$ spacing, wherein each position corresponds to a Bernoulli random variable with probability 0.001 to be a point scatterer. The resulting distribution is illustrated in Fig. 6(a) and 6(b), and it has a total of 85 point scatterers.

The 3D FFBP set up is identical to the previous scenario, and like before, the output image has $243 \times 243 \times 48$ voxels with a $0.05 \times 0.05 \times 0.3 \mathrm{~m}^{3}$ resolution. The $-6 \mathrm{~dB}$ isosurfaces are displayed in Fig. 6(c) and 6(d). Due to mutual interference between the point scatterers, their resulting amplitudes on the processed data are not uniform. So, the value $-6 \mathrm{~dB}$ was chosen rather than $-3 \mathrm{~dB}$ in order to provide a clearer depiction of all point scatterers.

Fig. 7 shows a plane section of the $3 \mathrm{D}$ FFBP and the BP output images. It can be noticed that the two figures are nearly identical, thus suggesting that both algorithms have been evenly affected by the added random phase error. The degree of coherence between the 3D FFBP and the BP output images is 0.9992 . The mean and the standard deviation of the phase error are $5 \times 10^{-5} \mathrm{rad}$ and $0.10 \mathrm{rad}$, respectively. The mean magnitude error is $0.1 \mathrm{~dB}$, and the standard deviation is 0.8 $\mathrm{dB}$. The processing time was 3.66 hours ( $\sim 0.15$ days) for the 3D FFBP and 39.71 hours $(\sim 1.7$ days) for the BP, which corresponds to a reduction of computation time of 10.8 times $(90.8 \%)$. A summary is provided in Table 3.

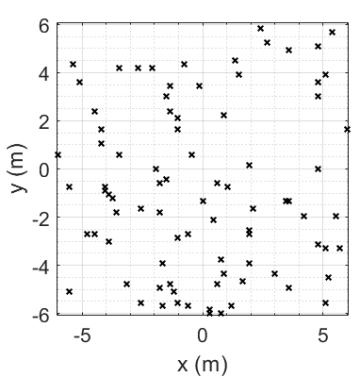

(a)

(c)

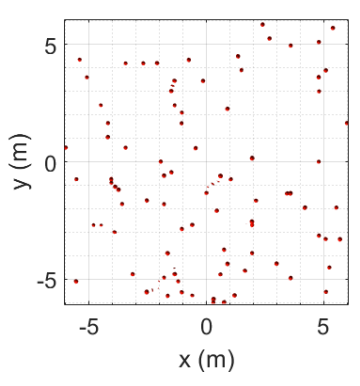

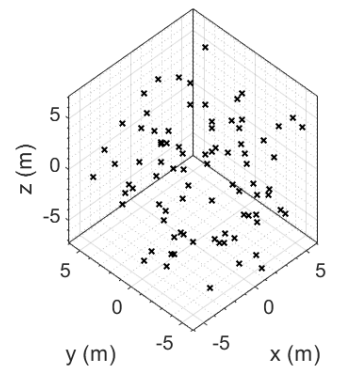

(b)

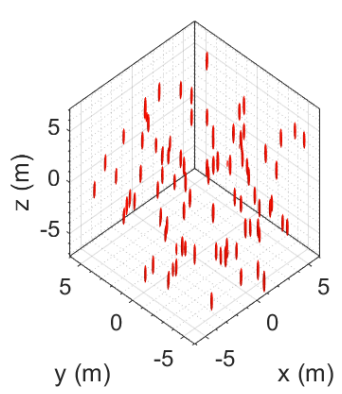

(d)
Fig. 6. Comparison between input and output target distributions: $(a, b)$ the input point scatterers and $(c, d)$ the $-6 \mathrm{~dB}$ isosurfaces for the 3D FFBP output image. The columns $(\mathrm{a}, \mathrm{c})$ and $(\mathrm{b}, \mathrm{d})$ show, respectively, the top and perspective views.

TABLE III. RANDOM POINT SCATTERERS - SiMULATION RESULTS

\begin{tabular}{|l|c|c|}
\hline \multicolumn{1}{|c|}{ Parameters } & Values & Units \\
\hline Mean phase error & $4 \times 10^{-5}$ & $\mathrm{rad}$ \\
\hline Standard deviation of the phase error & 0.10 & $\mathrm{rad}$ \\
\hline Mean magnitude error & 0.1 & $\mathrm{~dB}$ \\
\hline Standard deviation of the magnitude error & 0.8 & $\mathrm{~dB}$ \\
\hline Degree of coherence & 0.9992 & - \\
\hline Processing time - 3D FFBP & 3.66 & $\mathrm{~h}$ \\
\hline Processing time - BP & 39.71 & $\mathrm{~h}$ \\
\hline
\end{tabular}

\section{CONCLUSION}

The here presented 3D FFBP algorithm achieves shorter processing times than the direct BP, while still providing high-quality images for any flight path. McCorkle and Rofheart's quadtree approach was expanded by utilizing an adapted version of a traditional space-filling curve, called the Morton order curve $[8,9]$, for producing flexible 3D tree structures. The proposed algorithm also features an original method for generating sub-apertures based on a data mapping strategy, which is faster than recursively calculating weighted mean positions.

The 3D FFBP algorithm has been tested in two different simulation scenarios inspired by drone-borne SAR systems $[1,13]$ and data acquisition techniques that produce highresolution $3 \mathrm{D}$ images $[10,11]$. Tables 2 and 3 provide an outline of the simulation results. The 3 D FFBP achieved a $10-$ fold reduction in processing time without loss of image quality. Indeed, the degree of coherence between the $3 \mathrm{D}$ FFBP and BP images was nearly equal to one. In addition, 


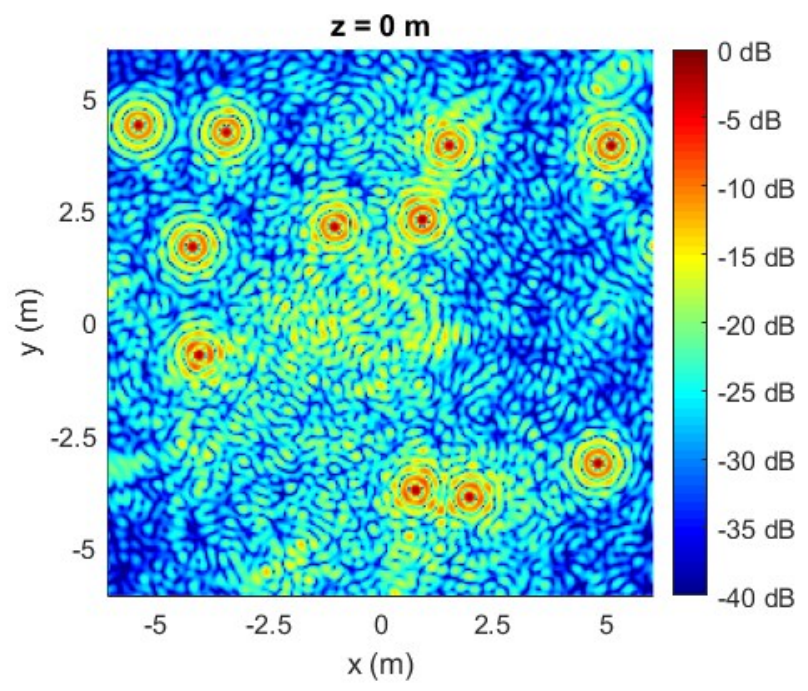

(a)

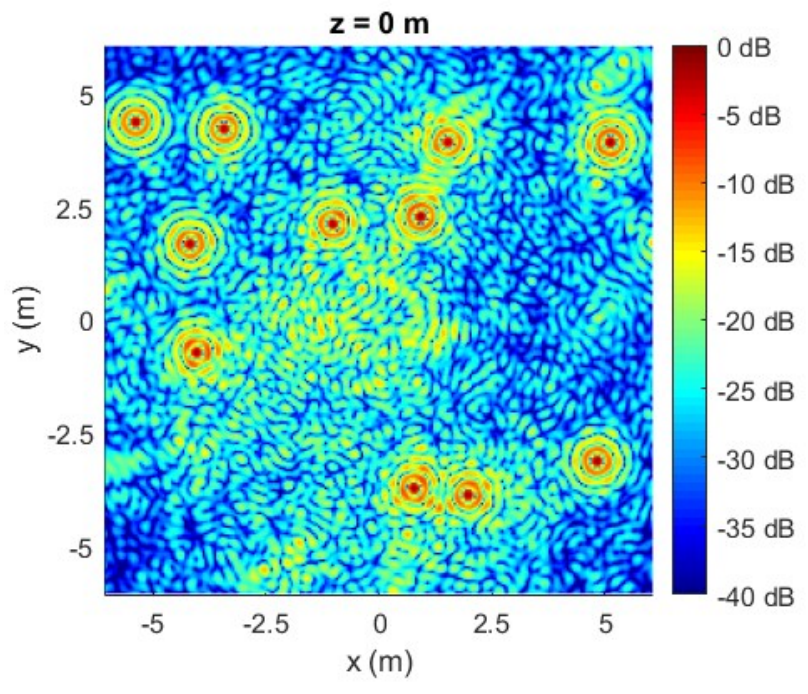

(b)

Fig. 7. Plane section for the $3 \mathrm{D}$ FFBP and BP results. Normalized magnitude in $\mathrm{dB}$ at $\mathrm{z}=0 \mathrm{~m}$ for (a) the $3 \mathrm{D}$ FFBP and (b) the BP output images.

the standard deviation of the phase error was considerably smaller than the typical $\pi / 8 \mathrm{rad}$ threshold used for assessing FFBP algorithms. The proposed sub-aperture definition method was $8 \%$ faster than the usual computation of weighted mean positions.

The time reduction could be further increased if another sampling strategy was used, possibly at the expense of a higher phase error. To take into account the spheres that circumscribe each sub-image helped to ensure excellent image quality, as the results have demonstrated. However, it is not a very efficient approach. In [12], instead of partitioning the range data, a fixed number of pivots is used for combining sub-apertures. The range data are processed all at once, using FFT interpolation to increase efficiency.

In future works, we shall improve the range sampling strategy to further enhance the 3D FFBP algorithm. Moreover, since drone-borne SAR systems seem to be a promising technology for performing SAR tomography, we shall continue exploring its suitability for this type of application.

\section{ACKNOWLEDGMENT}

The authors would like to thank João Roberto Moreira Neto and Luciano Prado de Oliveira for their support and assistance.

\section{REFERENCES}

[1] L. Moreira et al., "A drone-borne multiband DInSAR: Results and applications," in 2019 IEEE Radar Conference, RadarConf 2019, 2019.

[2] S. Dill, E. Schreiber, M. Engel, A. Heinzel, and M. Peichl, "A drone carried multichannel synthetic aperture radar for advanced buried object detection," in 2019 IEEE Radar Conference, RadarConf 2019, 2019.
[3] J. W. McCorkle and M. Rofheart, "Order $\mathrm{N}^{\wedge} 2 \log (\mathrm{N})$ backprojector algorithm for focusing wide-angle wide-bandwidth arbitrary-motion synthetic aperture radar," in Radar Sensor Technology, 1996, no. 2747, pp. 25-36.

[4] L. M. H. Ulander, H. Hellsten, and G. Stenström, "Synthetic-Aperture Radar Processing Using Fast Factorized Back-Projection," IEEE Trans. Aerosp. Electron. Syst., vol. 39, no. 3, pp. 760-776, Jul. 2003.

[5] O. Ponce et al., "Fully Polarimetric High-Resolution 3-D Imaging With Circular SAR at L-Band," IEEE Trans. Geosci. Remote Sens., vol. 52, no. 6, pp. 3074-3090, Jun. 2014.

[6] X. Song and W. Yu, "Processing video-SAR data with the fast backprojection method," IEEE Trans. Aerosp. Electron. Syst., vol. 52, no. 6, pp. 2838-2848, Dec. 2016.

[7] T. M. Marston, D. S. Plotnick, and K. L. Williams, "Three dimensional fast factorized back projection for sub-sediment imaging sonars," in OCEANS 2019 MTS/IEEE Seattle, OCEANS 2019, 2019.

[8] G. M. Morton, "A computer oriented geodetic data base and a new technique in the file sequencing," 1966.

[9] M. Connor and P. Kumar, "Fast construction of $\kappa$-nearest neighbor graphs for point clouds," IEEE Trans. Vis. Comput. Graph., vol. 16, no. 4, pp. 599-608, 2010.

[10] O. Ponce, P. Prats-Iraola, R. Scheiber, A. Reigber, and A. Moreira, "First Airborne Demonstration of Holographic SAR Tomography With Fully Polarimetric Multicircular Acquisitions at L-Band," IEEE Trans. Geosci. Remote Sens., vol. 54, no. 10, pp. 6170-6196, Oct. 2016.

[11] D. J. Sego, H. Griffiths, and M. C. Wicks, "Waveform and Aperture Design for Low-Frequency RF Tomography," IET Radar, Sonar Navig., vol. 5, no. 6, p. 686, 2011.

[12] H. Zhang, J. Tang, R. Wang, Y. Deng, W. Wang, and N. Li, “An Accelerated Backprojection Algorithm for Monostatic and Bistatic SAR Processing," Remote Sens., vol. 10, no. 1, p. 140, Jan. 2018.

[13] D. Luebeck et al., "Drone-borne Differential SAR Interferometry," Remote Sens., vol. 12, no. 5, p. 778, Feb. 2020.

[14] I. H. Woodhouse, Introduction to Microwave Remote Sensing, 1st ed. Speckled Press, 2015.

[15] E. J. Hughes and M. Leyland, "Using multiple genetic algorithms to generate radar point-scatterer models," IEEE Trans. Evol. Comput., vol. 4, no. 2, pp. 147-163, Jul. 2000. 